\title{
Weekly variation in health-care quality by day and time of admission: a nationwide, registry-based, prospective cohort study of acute stroke care
}

DOI:

10.1016/S0140-6736(16)30443-3

\section{Document Version \\ Accepted author manuscript}

Link to publication record in Manchester Research Explorer

Citation for published version (APA):

Bray, B. D., . Cloud, G. C., James, M. A., Hemingway, H., Paley, L., Stewart, K., Tyrrell, P., Wolfe, C. D. A., \& Rudd, A. G. (2016). Weekly variation in health-care quality by day and time of admission: a nationwide, registrybased, prospective cohort study of acute stroke care. The Lancet, 388(10040), 170-177. https://doi.org/10.1016/S0140-6736(16)30443-3

\section{Published in:}

The Lancet

\section{Citing this paper}

Please note that where the full-text provided on Manchester Research Explorer is the Author Accepted Manuscript or Proof version this may differ from the final Published version. If citing, it is advised that you check and use the publisher's definitive version.

\section{General rights}

Copyright and moral rights for the publications made accessible in the Research Explorer are retained by the authors and/or other copyright owners and it is a condition of accessing publications that users recognise and abide by the legal requirements associated with these rights.

\section{Takedown policy}

If you believe that this document breaches copyright please refer to the University of Manchester's Takedown Procedures [http://man.ac.uk/04Y6Bo] or contact uml.scholarlycommunications@manchester.ac.uk providing relevant details, so we can investigate your claim.

\section{OPEN ACCESS}




\title{
Weekly variation in health-care quality by day and time of admission: a nationwide, registry-based, prospective cohort study of acute stroke care
}

\author{
Benjamin D Bray, Geoffrey C Cloud, Martin A James, Harry Hemingway, Lizz Paley, Kevin Stewart, Pippa J Tyrrell, Charles D A Wolfe, \\ Anthony G Rudd, on behalf of the SSNAP collaboration
}

\section{Summary}

Background Studies in many health systems have shown evidence of poorer quality health care for patients admitted on weekends or overnight than for those admitted during the week (the so-called weekend effect). We postulated that variation in quality was dependent on not only day, but also time, of admission, and aimed to describe the pattern and magnitude of variation in the quality of acute stroke care across the entire week.

Methods We did this nationwide, registry-based, prospective cohort study using data from the Sentinel Stroke National Audit Programme. We included all adult patients (aged $>16$ years) admitted to hospital with acute stroke (ischaemic or primary intracerebral haemorrhage) in England and Wales between April 1, 2013, and March 31, 2014. Our outcome measure was 30 day post-admission survival. We estimated adjusted odds ratios for 13 indicators of acute stroke-care quality by fitting multilevel multivariable regression models across 42 4-h time periods per week.

Findings The study cohort comprised 74307 patients with acute stroke admitted to 199 hospitals. Care quality varied across the entire week, not only between weekends and weekdays, with different quality measures showing different patterns and magnitudes of temporal variation. We identified four patterns of variation: a diurnal pattern (thrombolysis, brain scan within $12 \mathrm{~h}$, brain scan within $1 \mathrm{~h}$, dysphagia screening), a day of the week pattern (stroke physician assessment, nurse assessment, physiotherapy, occupational therapy, and assessment of communication and swallowing by a speech and language therapist), an off-hours pattern (door-to-needle time for thrombolysis), and a flow pattern whereby quality changed sequentially across days (stroke-unit admission within $4 \mathrm{~h}$ ). The largest magnitude of variation was for door-to-needle time within $60 \mathrm{~min}$ (range in quality 35-66\% [16/46-232/350]; coefficient of variation 18.2). There was no difference in 30 day survival between weekends and weekdays (adjusted odds ratio 1.03, 95\% CI 0.95-1.13), but patients admitted overnight on weekdays had lower odds of survival $(0 \cdot 90,0 \cdot 82-0 \cdot 99)$.

Interpretation The weekend effect is a simplification, and just one of several patterns of weekly variation occurring in the quality of stroke care. Weekly variation should be further investigated in other health-care settings, and quality improvement should focus on reducing temporal variation in quality and not only the weekend effect.

Funding None.

\section{Introduction}

The quality of health care that patients receive might be partly determined by when they are admitted to hospital. ${ }^{1}$ The weekend effect (ie, poorer care quality and outcomes for patients admitted at the weekend than for those admitted during the week) or the off-hour effect (poorer care of patients admitted outside of usual working hours than of those admitted during regular hours) have been reported in many studies across a wide variety of clinical presentations. ${ }^{2-4}$ Such studies have had a major, and sometimes contentious, effect on health policy-eg, by prompting moves to increase the number of doctors working in hospitals at weekends. ${ }^{5}$ However, evidence for why health-care quality might be worse overnight or at the weekend is scarce and any understanding remains largely speculative, ${ }^{6}$ creating difficulty in health-policy development and quality improvement. Moreover, previous studies have generally compared weekdays with weekends, or regular hours with off-hours, rather than measuring care quality across both day of the week and time. This approach risks obscuring other patterns of temporal variation in care quality, which might have important implications for understanding and improving the quality of healthcare services.

We therefore aimed to describe the pattern and magnitude of weekly variation in several domains of care quality for people admitted to hospital with acute stroke. Globally, stroke is the second leading cause of death $^{7}$ and the third largest contributor to disease burden. ${ }^{8}$ There is good quality evidence for acute interventions (such as intravenous thrombolysis with alteplase and organised stroke-unit care) improving outcomes after stroke; ${ }^{9}$ how quickly acute stroke care is delivered is therefore both important and can be measured against evidence-based standards. We postulated that care quality is dependent not only on day of admission, but also time of admission.

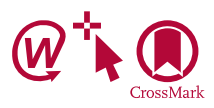

Published Online May 10, 2016 http://dx.doi.org/10.1016/ S0140-6736(16)30443-3

This online publication has been corrected. The corrected version first appeared at thelancet.com on May 17, 2016

See Online/Comment http://dx.doi.org/10.1016/ S0140-6736(16)30505-0 Farr Institute of Health Informatics Research, University College London, London, UK (B D Bray MD, Prof H Hemingway FRCP); St George's NHS Foundation Trust, London, UK (G C Cloud FRCP); Royal Devon and Exeter NHS Foundation Trust, Exeter, UK (M A James FRCP); Clinical Effectiveness and Evaluation Unit (K Stewart FRCP), Royal College of Physicians, London, UK (L Paley BA); Manchester Academic Health Sciences Centre, University of Manchester, Manchester, UK (Prof P J Tyrrell FRCP); Division of Health and Social Care Research, King's College London, London, UK (Prof C D A Wolfe FFPH); and Division of Health and Social Care Research, King's College London, London, UK (Prof A G Rudd FRCP) Correspondence to: Dr Benjamin D Bray, Farr Institute of Health Informatics Research, University College London, London NW1 2DA, UK benjamin.bray@kcl.ac.uk 
Research in context

\section{Evidence before this study}

Between Jan 1, 1980, and May 31, 2015, we searched MEDLINE for English-language studies published before June, 2015, investigating temporal variation in health-care quality. Our main focus was to identify studies of stroke care, but we also searched for studies done in other clinical settings. Our search terms were "Weekend", "Weekend effect", "Off hours", "Temporal variation", " AND Stroke", "AND quality". Studies of the weekend effect were identified in a wide range of clinical settings and geographical locations, describing evidence of poorer outcomes for patients admitted on the weekend or overnight with myocardial infarction, stroke, and general emergency admissions. We identified only a few studies that examined variation across both time of admission and day of week, including a study of obstetric outcomes in California, USA, and a study of hospital inpatients from Australia.

\section{Added value of this study}

Our study shows that in acute stroke care, the weekend effect is just one of several patterns of variation in quality that occur in real-world practice. Quality varied across the whole week, and different aspects of quality showed different patterns of variation.

\section{Implications of all the available evidence}

These findings imply that in acute stroke care, the weekend effect is a simplification of the true extent of temporal variation in health-care quality that occurs across the week. A focus only on reducing differences in care quality between weekends and weekdays will therefore not fully address the problem of variation in health-care quality across the week. Although we only examined care quality in the stroke setting, findings from previous studies assessing the weekend effect in a wide variety of clinical settings suggest that weekly variations in quality might also be pervasive across acute health-care settings. Such variations should be sought for and be a focus of quality improvement efforts.
For the SSNAP see https://www. strokeaudit.org

\section{Methods}

\section{Study design and patients}

We did this nationwide, registry-based, prospective cohort study using data from the Sentinel Stroke National Audit Programme (SSNAP) - the national register of stroke care in England and Wales. SSNAP collects data for the clinical characteristics and care quality (measuring various aspects of care from the time of admission up to 6 months after stroke) of patients admitted to all acutecare hospitals in England and Wales with acute ischaemic stroke or primary intracerebral haemorrhage. Data were collected prospectively and validated by clinical teams and entered into the SSNAP database via a secure web interface. We used an anonymised extract of this database. SSNAP is estimated to include roughly $95 \%$ of all adults admitted to hospital in England and Wales with stroke. The study cohort included all adult patients (aged $>16$ years) admitted to hospital with acute stroke (ischaemic or primary intracerebral haemorrhage) in England and Wales between April 1, 2013, and March 31, 2014.

SSNAP has approval from the Confidentiality Advisory Group of the Health Research Authority to collect patient data under section 251 of the National Health Service Act 2006. No additional ethical approval was sought.

\section{Procedures}

We measured care quality using a pre-existing set of quality indicators reported routinely by SSNAP, which are derived from UK national guidelines.' These indicators reflect the time-critical nature of acute stroke care: receiving a brain scan within $1 \mathrm{~h}$ or $12 \mathrm{~h}$ of admission; direct admission to a stroke unit (or intensive care or high-dependency unit) within $4 \mathrm{~h}$; administration of intravenous thrombolysis with alteplase; door-toneedle time of less than $60 \mathrm{~min}$ for patients treated with alteplase; dysphagia screening within $4 \mathrm{~h}$; reviews by a stroke specialist physician and nurse within $24 \mathrm{~h}$; and assessments by physiotherapy, occupational therapy, and speech and language therapy within $72 \mathrm{~h}$. Patients with clinical exclusions for dysphagia screening or therapy assessments (eg, those being treated palliatively only) were excluded from the denominator of these specific indicators. Only patients with ischaemic stroke presenting within $4.5 \mathrm{~h}$ of stroke onset were included in the denominator for thrombolysis.

\section{Statistical analysis}

Our outcome measure was 30 day post-admission survival. We did time-stratified analyses by classifying patients according to time of admission. We used time of stroke onset for patients who had stroke while in hospital. We used two methods for stratifying time. First, we used six, $4 \mathrm{~h}$ time blocks per day of the week $(0000 \mathrm{~h}$ to $0359 \mathrm{~h}$, $0400 \mathrm{~h}$ to $0759 \mathrm{~h}, 0800 \mathrm{~h}$ to $1159 \mathrm{~h}, 1200 \mathrm{~h}$ to $1559 \mathrm{~h}$, $1600 \mathrm{~h}$ to $1959 \mathrm{~h}$, and $2000 \mathrm{~h}$ to $2359 \mathrm{~h}$ ), resulting in 42 time categories in total. We chose $4 \mathrm{~h}$ periods because they were the shortest time periods that provided sufficient numbers of patients in each block for model fitting. Second, we used larger time periods corresponding to weekends and weekdays and regular hours or off-hours to aid comparison with previous literature of weekend effects: Monday to Friday 0800-1959 h, Saturday to Sunday 0800-1959 h, Monday to Friday 2000-0759 h, and Saturday to Sunday $2000-0759 \mathrm{~h}$. 


\begin{tabular}{|lc}
\hline & Summary statistics \\
\hline Nex & 74307 \\
Male & \\
Female & $36873(50 \%)$ \\
Age (years) & $37434(50 \%)$ \\
Stroke type & $77(67-85)$ \\
Ischaemic & \\
Intracerebral haemorrhage & $65193(88 \%)$ \\
Undetermined & $8038(11 \%)$ \\
Pre-stroke modified Rankin Scale score & $1076(2 \%)$ \\
0 & \\
1 & $42524(57 \%)$ \\
2 & $11311(15 \%)$ \\
3 & $7011(9 \%)$ \\
4 & $7801(11 \%)$ \\
5 & $4249(6 \%)$ \\
NIHSS score on arrival & $1391(2 \%)$ \\
Level of consciousness on arrival & $4(2-10)$ \\
0 (alert) & \\
1 (not alert; responds to voice) & $61638(83 \%)$ \\
2 (not alert; responds to pain) & $7482(10 \%)$ \\
3 (totally unresponsive) & $2978(4 \%)$ \\
Comorbidity & $2209(3 \%)$ \\
Heart failure & \\
Hypertension & $4079(6 \%)$ \\
Atrial fibrillation & $39918(54 \%)$ \\
Diabetes mellitus & $15385(21 \%)$ \\
Previous stroke or transient ischaemic attack & $20292(27 \%)$ \\
& $($ Table 1 continues in next column) \\
&
\end{tabular}

We quantified the magnitude of variation in care quality between time blocks by calculating the coefficient of variation (the ratio of the SD to the mean, multiplied by 100). We used the coefficient of variation because it allows the dispersion of variables with different means to be compared.

We did multivariable analysis by fitting multilevel ${ }^{10}$ logistic regression models, including patient age, sex, place of stroke onset (in or out of hospital), stroke type, vascular comorbidity (atrial fibrillation, heart failure, diabetes mellitus, previous stroke or transient ischaemic attack, hypertension), pre-stroke functional level (as measured by the modified Rankin Scale ${ }^{11}$ ), time from stroke onset to admission, stroke severity (National Institutes of Health Stroke Scale score [NIHSS] or level of consciousness on admission) and hospital-level random intercepts. We included time categories as fixed effects. We used the middle-ranking time period (21st) in the unadjusted analyses as the reference category in the models using 42 time blocks per week, and used Monday to Friday $0800-1959 \mathrm{~h}$ as the reference category in the models using four time blocks per week. We calculated adjusted absolute effect sizes using marginal standardisation. ${ }^{12}$

\begin{tabular}{|c|c|}
\hline & Summary statistics \\
\hline \multicolumn{2}{|c|}{ (Continued from previous column) } \\
\hline Onset in hospital & $3969(5 \%)$ \\
\hline \multicolumn{2}{|l|}{ Time from onset to admission } \\
\hline Unclear symptom onset* & $28739(39 \%)$ \\
\hline$<180 \min$ & $25441(34 \%)$ \\
\hline $180-359 \mathrm{~min}$ & $7126(10 \%)$ \\
\hline$\geq 360 \mathrm{~min}$ & $13001(18 \%)$ \\
\hline \multicolumn{2}{|l|}{ Day of admission } \\
\hline Sunday & $9515(13 \%)$ \\
\hline Monday & $11618(16 \%)$ \\
\hline Tuesday & $11077(15 \%)$ \\
\hline Wednesday & $11058(15 \%)$ \\
\hline Thursday & $10882(15 \%)$ \\
\hline Friday & $10756(15 \%)$ \\
\hline Saturday & $9401(13 \%)$ \\
\hline \multicolumn{2}{|c|}{ Day of discharge if discharged alive } \\
\hline Sunday & $1955(3 \%)$ \\
\hline Monday & $10701(17 \%)$ \\
\hline Tuesday & $11467(18 \%)$ \\
\hline Wednesday & $11012(18 \%)$ \\
\hline Thursday & $11061(18 \%)$ \\
\hline Friday & $13268(21 \%)$ \\
\hline Saturday & $3578(6 \%)$ \\
\hline Alive at 30 days & $64597(87 \%)$ \\
\hline \multicolumn{2}{|c|}{$\begin{array}{l}\text { Data are } \mathrm{n}(\%) \text { or median (IQR), unless otherwise specified. NIHSS=National } \\
\text { Institutes of Health Stroke Scale. }{ }^{*} \text { Eg, wake-up stroke. }\end{array}$} \\
\hline
\end{tabular}

We did sensitivity analyses to explore the effect of data missing for NIHSS on admission. First, models were fitted with level of consciousness on admission as a proxy for stroke severity, and the results compared with models using NIHSS. Second, models were fitted after multiple imputation ${ }^{13}$ of 20 datasets. Sensitivity analyses were also done after exclusion of patients who died within 1 day of admission. Analyses and visualisations were done with Stata (version 14).

\section{Role of the funding source}

There was no funding source for this study. The corresponding author had full access to all the data in the study and had final responsibility for the decision to submit for publication.

\section{Results}

The study cohort comprised 74307 patients with acute stroke admitted to 199 hospitals. The median age of patients was 77 years (IQR 67-85) and 65193 (88\%) patients had an ischaemic stroke (table 1). The most frequent day of admission was Monday, and admissions were less frequent on Saturdays and Sundays than on weekdays (table 1). Discharges from hospital were less common at weekends than during the week (table 1). Data were 100\% complete 
for all baseline variables apart from NIHSS on admission, which was available for 54048 (73\%) patients.

The magnitude and pattern of temporal variation in quality varied widely across the 13 quality indicators (table 2). In unadjusted analyses, we recorded the greatest magnitude of variation for door-to-needle time of less than $60 \mathrm{~min}$ (table 2). The indicators with the smallest variation were 30 day survival and assessment by a stroke nurse within $24 \mathrm{~h}$ (table 2).

We noted four main patterns of temporal variation in heat maps (figures 1, 2, 3, 4), and these were similar in See Online for appendix both the unadjusted and multivariable analyses of each

\begin{tabular}{|c|c|c|c|}
\hline & Range in quality* & Mean (SD) & $\begin{array}{l}\text { Coefficient } \\
\text { of variation }\end{array}$ \\
\hline Thrombolysis rate $\dagger$ & $21-37 \%(38 / 179-76 / 205)$ & $32 \cdot 1(3.9)$ & $12 \cdot 6$ \\
\hline Door-to-needle time $<60 \mathrm{~min}$ & $35-66 \%(16 / 46-232 / 350)$ & $49 \cdot 1(8 \cdot 9)$ & $18 \cdot 2$ \\
\hline Brain scan within $1 \mathrm{~h}$ & $34-47 \%(186 / 543-1403 / 2980)$ & $41 \cdot 7(2 \cdot 8)$ & $6 \cdot 6$ \\
\hline Brain scan within $12 \mathrm{~h}$ & $72-95 \%(1815 / 2510-2837 / 2980)$ & $84 \cdot 0(7 \cdot 3)$ & $8 \cdot 7$ \\
\hline Stroke-unit admission within $4 \mathrm{~h}$ & $46-65 \%(293 / 607-2026 / 3086)$ & $56 \cdot 4(4 \cdot 5)$ & 8.0 \\
\hline Dysphagia screen within $4 \mathrm{~h}$ & $50-73 \%(249 / 495-1911 / 2624)$ & $61 \cdot 5(5 \cdot 8)$ & $9 \cdot 4$ \\
\hline Stroke physician within $24 \mathrm{~h}$ & $49-85 \%(266 / 543-1148 / 1351)$ & $71.8(9 \cdot 8)$ & $13 \cdot 6$ \\
\hline Stroke nurse within $24 \mathrm{~h}$ & $77-90 \%(394 / 509-2784 / 3086)$ & $85 \cdot 4(3.0)$ & $3 \cdot 5$ \\
\hline Physiotherapy assessment within $72 \mathrm{~h}$ & $81-97 \%(363 / 447-551 / 566)$ & $93.0(3.9)$ & $4 \cdot 2$ \\
\hline Occupational therapy assessment within $72 \mathrm{~h}$ & $71-92 \%(293 / 415-1830 / 1998)$ & $85.8(5.4)$ & $6 \cdot 3$ \\
\hline $\begin{array}{l}\text { Communication assessment by speech and } \\
\text { language therapist within } 72 \mathrm{~h}\end{array}$ & $50-89 \%(620 / 1253-623 / 700)$ & $77 \cdot 4(8 \cdot 9)$ & $11 \cdot 5$ \\
\hline $\begin{array}{l}\text { Swallowing assessment by speech and } \\
\text { language therapist within } 72 \mathrm{~h}\end{array}$ & $63-87 \%(749 / 1184-263 / 301)$ & $78 \cdot 3(5 \cdot 6)$ & $7 \cdot 2$ \\
\hline 30 day survival & $80-90 \%(432 / 543-2918 / 3252)$ & $85 \cdot 9(2 \cdot 6)$ & $3 \cdot 1$ \\
\hline \multicolumn{4}{|c|}{$\begin{array}{l}\text { Data are } n / N \text {, unless otherwise specified. *From lowest to highest time category. †Of patients with ischaemic stroke } \\
\text { presenting within } 4.5 \text { h of stroke onset. }\end{array}$} \\
\hline
\end{tabular}

indicator. Four of the indicators showed a diurnal pattern of variation, with quality varying across time of day (thrombolysis, brain scan within $12 \mathrm{~h}$, brain scan within $1 \mathrm{~h}$, dysphagia screening within $4 \mathrm{~h}$; figures 1 , 2). This variation was not only restricted to differences between daytime and overnight admissions-for example, patients arriving in the morning were more likely to receive a brain scan within $1 \mathrm{~h}$ than were those admitted in the afternoon (figure 1). Six of the indicators varied across days of the week, with lower quality care for weekend admissions (stroke physician assessment and nurse assessment within $24 \mathrm{~h}$; figure 2) or for patients admitted on a Thursday or Friday (physiotherapy, occupational therapy, and assessment of communication and swallowing by a speech and language therapist within $72 \mathrm{~h}$; figure 3 ). The third pattern was for poorer care both overnight and at the weekend (door-to-needle time $<60 \mathrm{~min}$ for thrombolysis; figure 1). The fourth pattern was of sequential change in quality across both day and time, with quality improving sequentially across weekdays and then deteriorating at the weekend, resulting in patients on Mondays having the lowest odds of being admitted to a stroke unit within $4 \mathrm{~h}$ (figure 2).

There was no difference in adjusted 30 day survival between patients admitted during the day at the weekend and those admitted on weekdays in the models using either NIHSS (adjusted odds ratio [OR] 1.03, 95\% CI 0.95-1.13) or level of consciousness $(0.97,0.91-1.04)$, but weak evidence that survival was worse for patients admitted overnight on weekdays (adjusted OR $0.90,95 \% \mathrm{CI}$ $0 \cdot 82-0 \cdot 99$; absolute difference in adjusted survival $-0 \cdot 7 \%$, $-1 \cdot 2$ to $-0 \cdot 2$; table 3 , appendix). The point estimate and confidence intervals of survival for patients admitted

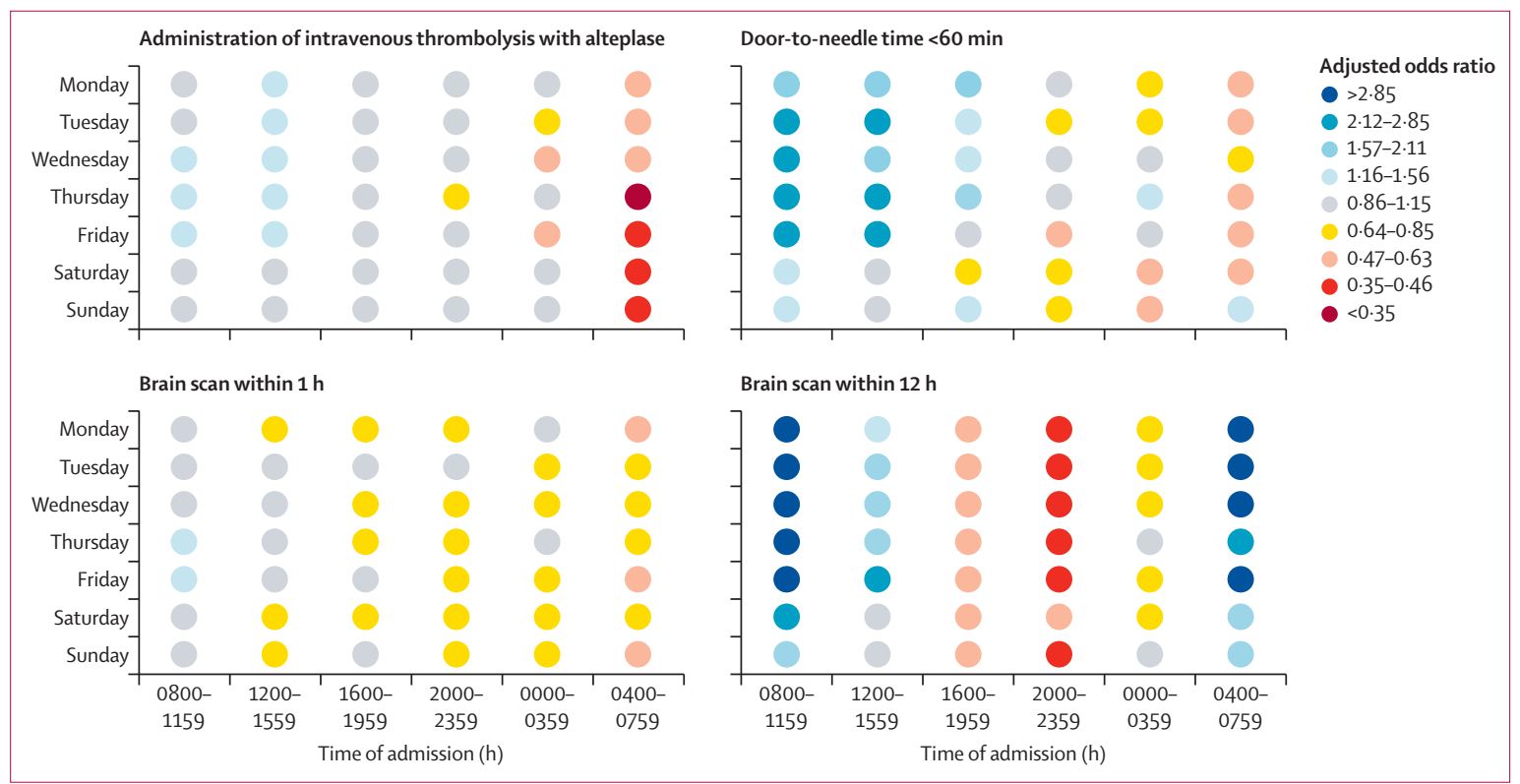

Figure 1: Variations in administration of thrombolysis, door-to-needle time less than $60 \mathrm{~min}$, brain scan within $1 \mathrm{~h}$, and brain scan within $12 \mathrm{~h}$ 


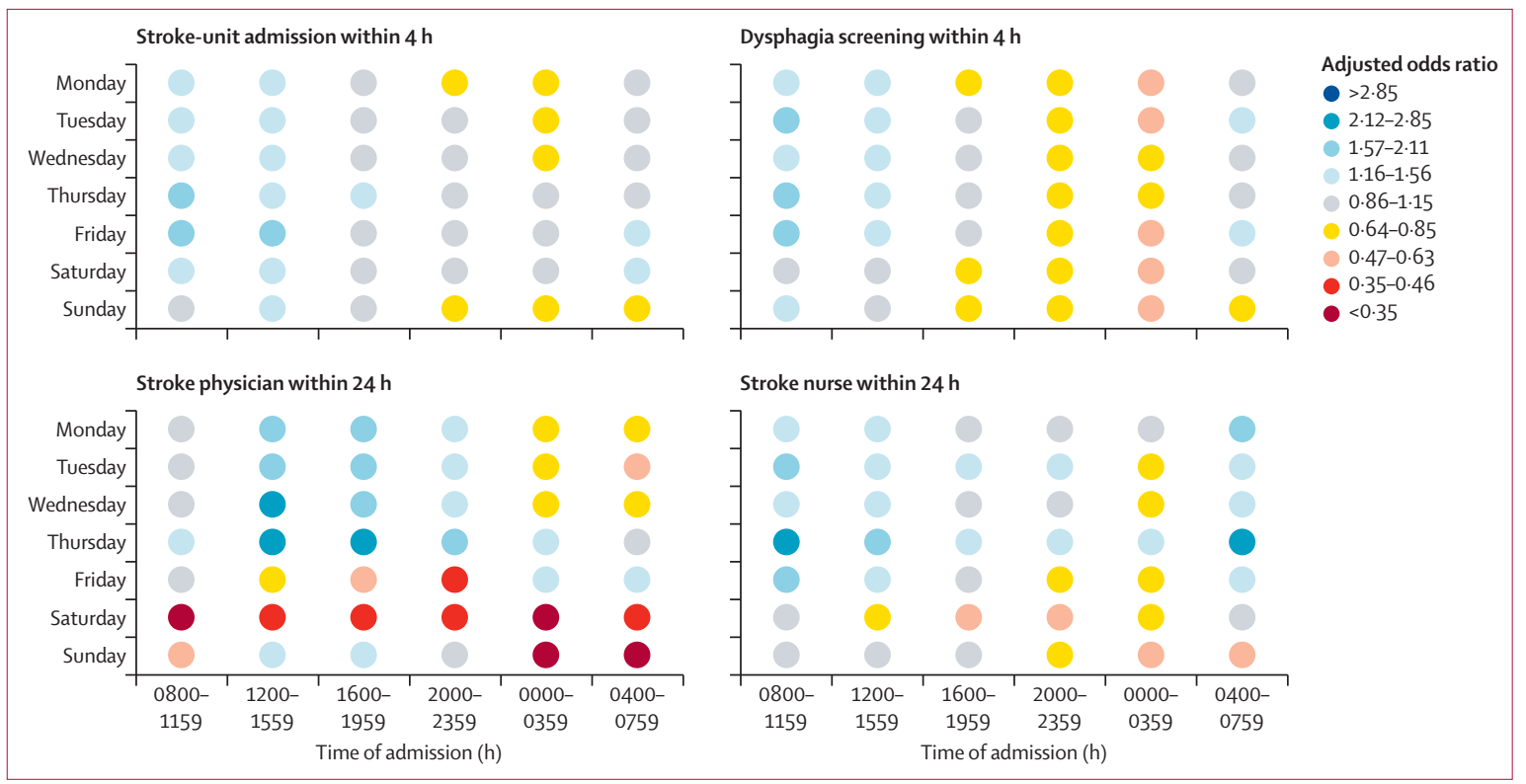

Figure 2: Variations in stroke-unit admission within $4 \mathrm{~h}$, dysphagia screening within $4 \mathrm{~h}$, stroke physician within $24 \mathrm{~h}$, and stroke nurse within $24 \mathrm{~h}$

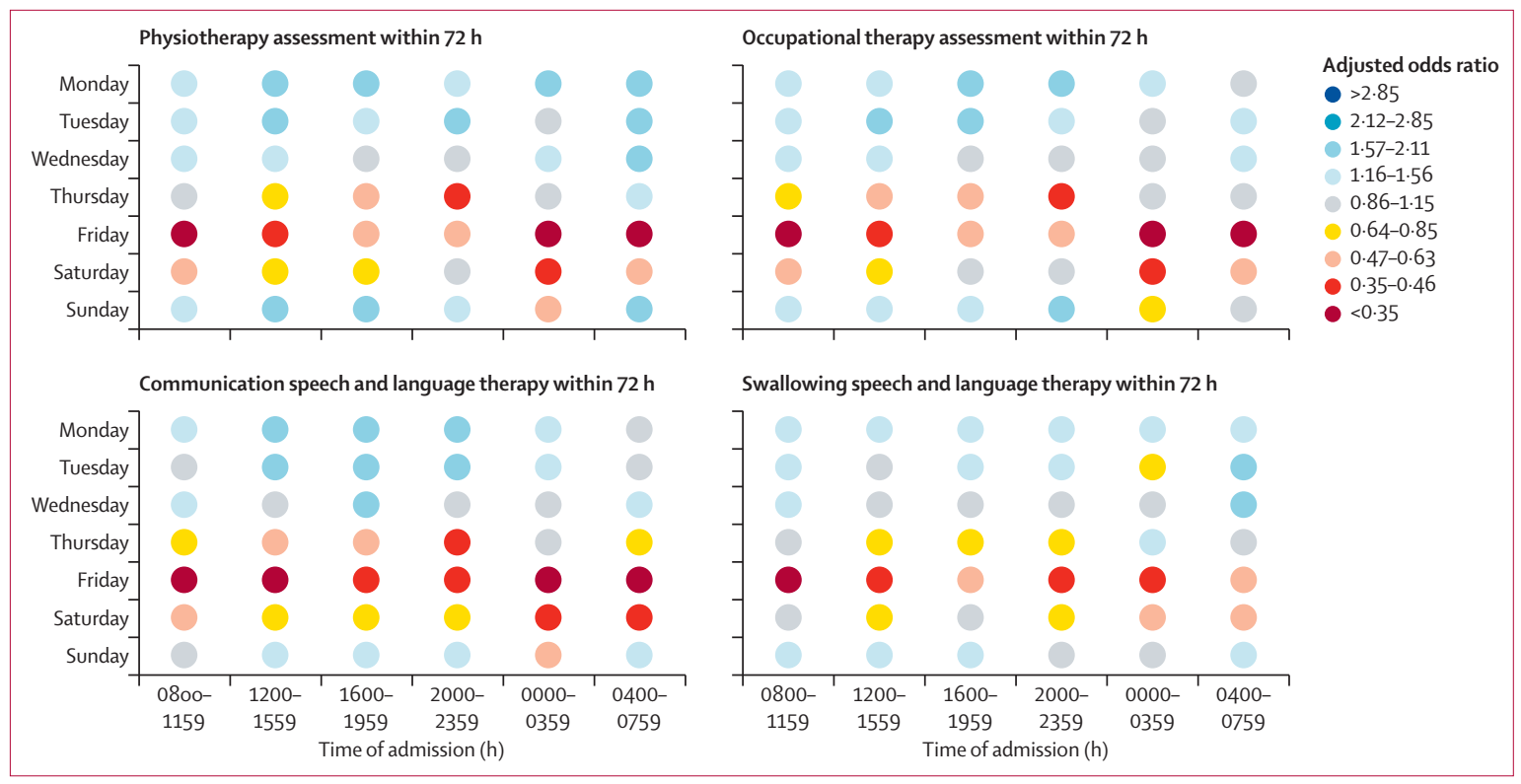

Figure 3: Variations in physiotherapy assessment within $72 \mathrm{~h}$, occupational therapy assessment within $72 \mathrm{~h}$, communication speech and language therapy assessment within $72 \mathrm{~h}$, and swallowing speech and language therapy assessment within $72 \mathrm{~h}$

overnight at weekends differed between models: survival was poorer in the models using level of consciousness (adjusted OR 0.84, 95\% CI 0.77-0.93; absolute difference $-1 \cdot 5 \%,-2 \cdot 3 \%$ to $-0 \cdot 7 \%$ ) and with multiply imputed NIHSS data $(0 \cdot 86,0 \cdot 77-0 \cdot 95)$, but not in the model using NIHSS $(0 \cdot 89,0 \cdot 78-1 \cdot 01 ;$ appendix). The sensitivity analyses using imputed datasets and excluding patients who died within 1 day of admission were otherwise similar; the only change of note in the analysis of patients who died within 1 day was a modest reduction in effect size for brain scanning within $1 \mathrm{~h}$ (appendix).

\section{Discussion}

Our study shows that variations in the quality of acute stroke care happen across the whole week and not only between weekends and weekdays, with individual indicators of care quality differing in the magnitude and pattern of variation. This finding suggests that even within a single, well-defined clinical pathway such as acute stroke care, temporal variation is a complex occurrence that probably has various causes. Our findings show that the concept of the weekend effect is a major simplification of the true extent and nature of 


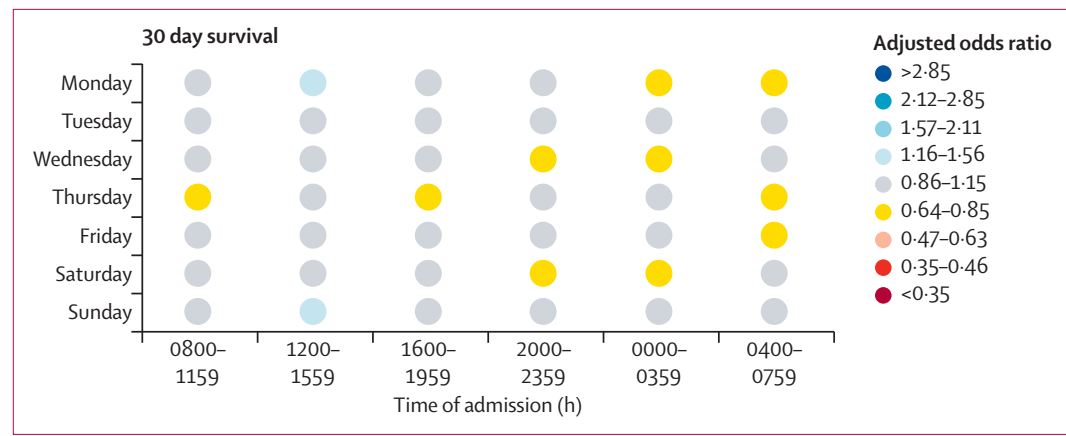

Figure 4: Variations in 30 day survival

\begin{tabular}{|c|c|c|c|c|}
\hline & $\begin{array}{l}\text { Weekday } \\
0800-1959 h^{*}\end{array}$ & $\begin{array}{l}\text { Weekend } \\
0800-1959 \mathrm{~h}\end{array}$ & $\begin{array}{l}\text { Weekday } \\
2000-0759 \mathrm{~h}\end{array}$ & $\begin{array}{l}\text { Weekend } \\
\text { 2000-0759 h }\end{array}$ \\
\hline Thrombolysis & 1 & $0.86(0.79-0.95)$ & $0.67(0.61-0.74)$ & $0.73(0.64-0.84)$ \\
\hline Door-to-needle time $<60 \mathrm{~min}$ & 1 & $0.55(0.47-0.63)$ & $0.40(0.34-0.46)$ & $0.35(0.28-0.43)$ \\
\hline Brain scan within $1 \mathrm{~h}$ & 1 & $0.83(0.78-0.87)$ & $0.76(0.72-0.80)$ & $0.72(0.66-0.78)$ \\
\hline Brain scan within $12 \mathrm{~h}$ & 1 & $0.76(0.70-0.81)$ & $0.51(0.47-0.55)$ & $0.51(0.45-0.57)$ \\
\hline $\begin{array}{l}\text { Stroke-unit admission within } \\
4 \mathrm{~h}\end{array}$ & 1 & $0.78(0.74-0.83)$ & $0.71(0.67-0.75)$ & $0.67(0.61-0.73)$ \\
\hline $\begin{array}{l}\text { Dysphagia screening within } \\
4 \mathrm{~h}\end{array}$ & 1 & $0.75(0.71-0.79)$ & $0.61(0.58-0.65)$ & $0.55(0.50-0.60)$ \\
\hline Stroke physician within $24 \mathrm{~h}$ & 1 & $0.42(0.40-0.45)$ & $0.77(0.72-0.82)$ & $0.34(0.31-0.37)$ \\
\hline $\begin{array}{l}\text { Specialist stroke nurse within } \\
24 \mathrm{~h}\end{array}$ & 1 & $0.63(0.58-0.68)$ & $0.80(0.73-0.88)$ & $0.48(0.42-0.54)$ \\
\hline $\begin{array}{l}\text { Physiotherapy assessment } \\
\text { within } 72 \mathrm{~h}\end{array}$ & 1 & $1.25(1.11-1.40)$ & $0.95(0.85-1.07)$ & $1.00(0.84-1.19)$ \\
\hline $\begin{array}{l}\text { Occupational therapy } \\
\text { assessment within } 72 \mathrm{~h}\end{array}$ & 1 & $1.18(1.08-1.29)$ & $0.94(0.87-1.03)$ & $1.03(0.90-1.18)$ \\
\hline $\begin{array}{l}\text { Communication assessment } \\
\text { by speech and language } \\
\text { therapist within } 72 \mathrm{~h}\end{array}$ & 1 & $1.25(1.14-1 \cdot 37)$ & $1.09(0.99-1.20)$ & $1.05(0.91-1.22)$ \\
\hline $\begin{array}{l}\text { Swallowing assessment by } \\
\text { speech and language } \\
\text { therapist within } 72 \mathrm{~h}\end{array}$ & 1 & $1 \cdot 10(1 \cdot 00-1 \cdot 23)$ & $1.04(0.94-1.16)$ & $0.94(0.80-1.11)$ \\
\hline 30 day survival & 1 & $1.03(0.95-1.13)$ & $0.90(0.82-0.99)$ & $0.89(0.78-1.01)$ \\
\hline
\end{tabular}

Data are odds ratio $(95 \% \mathrm{Cl})$. Multivariable model including stroke severity (National Institutes of Health Stroke Scale) age, sex, stroke type, place of stroke onset, pre-stroke level of functioning, vascular comorbidity, elapsed time from stroke onset to admission, and hospital-level random intercepts. *Reference category.

Table 3: Adjusted odds ratios of receiving each care-quality indicator

temporal variation in health-care quality, and that it is just one of several patterns of variation in care quality that occur in real-world clinical practice. Unmasking these potentially hidden sources of variation in quality through appropriate data collection and visualisation might aid identification of the factors causing variation in quality (such as staffing levels or bed capacity), and could be an important tool for quality improvement in health care.

Extensive literature exists exploring differences in care quality and outcomes between weekdays and weekends. $^{2-4,14,15}$ Some studies have also described differences in care between daytimes and overnight ${ }^{16}$ and between regular hours and off-hours. ${ }^{17}$ Findings from studies of the weekend effect in stroke care specifically have been conflicting. Some have shown evidence of reduced care quality (but no difference in mortality) for patients admitted on weekends, ${ }^{18}$ and the evidence for differences in mortality between weekend and weekday admissions is mixed. ${ }^{19-21}$ These differences might be explained by differences in how stroke-care services are organised, ${ }^{21}$ and some evidence shows that low nurse staffing levels on stroke units are associated with increased mortality at weekends. ${ }^{22} \mathrm{~A}$ limitation of much of the previous literature is that it has typically been based on comparisons of weekends versus weekdays or regular versus off-hours, without taking into account variation that might occur across both day of the week and time of day. However, a few studies have investigated how care might vary in this way. For example, administrative data have been used to model daily and diurnal patterns in mortality risk as part of a prognostic model for hospital inpatients, ${ }^{23}$ and identified weekend effects lagging into the following week. ${ }^{24}$ Diurnal patterns have also been observed in the frequency of obstetric complications..$^{25}$ Therefore, the patterns of health-care quality observed in our study are probably not restricted to stroke care, and might be found in other acute healthcare settings if they were sought for.

We identified four main patterns of temporal variation in stroke-care quality and postulate that they reflect differing underlying causal factors. Our study is not able to identify what these causal factors are, but might generate hypotheses for future studies. Establishment of characteristic patterns of variation might be useful to help identify and tackle these underlying causes, and thus to organise health-care services more effectively. The diurnal patterns we recorded might be the result of reduced clinical services overnight, such as lower staffing levels or reduced access to diagnostics. However, variation in quality also occurred during usual working hours, suggesting that other contributory factors might exist. For example, that patients admitted in the afternoon were less likely to receive an urgent brain scan than those admitted in the morning might be due to higher demand for CT scanning at busier times of the day.

Variation in quality that relates directly to admission on, or in relation to, the weekend suggests that how health care is organised on the weekend affects quality. Survey data show that stroke services in England and Wales are more likely to provide 7 day physiotherapy than occupational therapy or speech therapy services, ${ }^{10}$ which is consistent with the pattern of variation noted in this study. The data also show that provision of health care on weekends might affect patients admitted on other days of the week, with patients admitted on Thursdays and Fridays having the longest waits for therapy assessment.

One indicator (door-to-needle time) had a strong association with both day of week and time of day, with reduced performance both overnight and at weekends. Achievement of fast door-to-needle times in acute stroke requires that the entire diagnostic, decision-making, and 
treatment pathway is undertaken quickly; if just one stage is slow, this could cause serious delays in the whole pathway. Interventions that require this type of rapid, coordinated, systems response with on-site presence of key decision makers might therefore show the greatest magnitude of weekly variation. The pattern of care quality for stroke-unit access seems most likely to reflect patient flow and bed capacity within stroke-care services. We believe this pattern could be due to loss of spare bed capacity over the weekend as a result of reduced frequency of hospital discharges, resulting in the slowest transfers to stroke units occurring on Mondays.

Variation in survival after stroke was largely explained by differences in patient characteristics, with proportionally more unwell patients being admitted during off-hours. Therefore, one of the reasons for apparent temporal variation in care quality is factors that determine when and how patients present to health-care services. The conflicting nature of the literature on the presence or not of the weekend effect might reflect the ability of different studies to control for this source of confounding. ${ }^{26}$

Further research could help to test these hypotheses and identify the reasons for these patterns of temporal variation, identify new patterns of temporal variation, and perhaps aid in development of new taxonomies of temporal variation in health-care quality. In the meantime, our findings imply that there will not be one solution to eradication of time-based inequalities in care. Solutions are likely to require not only actions to ensure appropriate clinical staffing, but also measures to improve the capacity and use of beds, generate efficient patient flow, improve access to diagnostic and clinical support services, and improve the overall resilience of care pathways. Solutions should also consider the wider health-care system and not only the hospital in isolation, such as the availability of social care and community services at the weekends, on which patient discharges from hospital are dependent. Much of the current discourse on reducing weekend effects has occurred in the absence of a detailed understanding of why temporal variation in care quality occurs. Because solutions are likely to come at substantial financial and opportunity cost, ${ }^{27}$ policy makers, healthcare managers, and funders need to ensure that the reasons for temporal variation in quality are properly understood and that resources are targeted appropriately. For example, simply transferring clinicians from weekdays to weekends might not have the intended effect on quality and could lead to unintended consequences for the quality of care provided on weekdays. One potential method for gaining a better insight into variations in care quality might be to make use of the types of data visualisations we have used in this study, which is becoming increasingly feasible as electronic health-care data increase in scope and detail.

Our study has some limitations. Overall the data were very complete and strengthened by being from a national registry of clinical, rather than administrative, data; however, data were missing for one variable. Although the main analysis used a complete-case analysis, study results were similar when a proxy measure was used, and when multiple imputation was used to account for missing data. We measured outcomes using survival, which, although important, is a relatively restricted measure of stroke outcomes. The study would have been strengthened by use of other measures such as disability and quality of life. Nonetheless, most of the process measures used have a strong empirical rationale from randomised controlled trial evidence, ${ }^{28,29}$ and long-term disability data are not currently available in SSNAP. There was little similarity in the pattern of variation between survival and the other quality measures, which might be because these interventions do not affect survival (eg, thrombolysis with alteplase reduces disability but not mortality ${ }^{28}$ ) or because association exists at the patient level but not at the group level. We used time-sensitive indicators of care quality, which are likely to be more subject to temporal variation than aspects of care for which timeliness is less important. However, use of these indicators was not arbitrary, and we used the already existing national set of acute stroke indicators. We used the relatively simple method of stratifying by time rather than fitting more complex timeseries models; this method has the disadvantage of assuming that time changes in blocks rather than continuously. In future studies, we plan to explore different methods to model the effect of day of week and time of day, and to use larger datasets to reduce the time resolution to shorter time periods.

In summary, we found evidence that care quality in acute stroke care varies with time in more complex ways than previous studies of the weekend effect in health care would suggest. Although this study is of the quality of care received by people with acute stroke, it seems unlikely that stroke care alone displays such patterns of temporal variation in quality. Extension of this methodology to other areas of health care would be useful further areas of research, particularly for presentations for which the timeliness of care is an important determinant of outcomes (such as acute myocardial infarction or surgical emergencies). Finally, a more sophisticated understanding of the patterns of and reasons for temporal variation in care quality is needed, and this should become a routine part of quality improvement in health care.

\section{Contributors}

BDB devised the study, did the analysis, and wrote the manuscript. GCC, MAJ, PJT, and AGR wrote the manuscript, and provided clinical insight and critical commentary. $\mathrm{HH}, \mathrm{KS}$, and CDAW wrote the manuscript and provided critical commentary. LP did the analysis and wrote the manuscript. Boehringer Ingelheim, outside the submitted work. PJT is Trustee and medical Vice Chair of the Stroke Association, and Associate Director of the Royal College of Physicians Stroke Programme. All other authors declare no competing interests. 


\section{Acknowledgments}

The Sentinel Stroke National Audit Programme (SSNAP) is funded by the Healthcare Quality Improvement Partnership on behalf of National Health Service (NHS) England. The study received no specific funding, but some of the authors are funded either fully or partly. BDB is supported by the National Institute for Health Research (NIHR) as an academic clinical fellow. MAJ is supported by the NIHR Collaboration for Leadership in Applied Health Research and Care for the South West Peninsula. CDAW acknowledges financial support from the NIHR Biomedical Research Centre based at Guy's and St Thomas' NHS Foundation Trust and King's College London, the Stanley Thomas Johnson Foundation, the Stroke Association, and NIHR Programme Grant funding. HH is supported by awards establishing the Farr Institute of Health Informatics Research from the Medical Research Council (reference MR/K006584/1), in partnership with Arthritis Research UK, the British Heart Foundation, Cancer Research UK, the Economic and Social Research Council, the Engineering and Physical Sciences Research Council, NIHR, the National Institute for Social Care and Health Research (Welsh Assembly Government), the Chief Scientific Office (Scottish Government Health Directorates), and the Wellcome Trust. We thank the many hundreds of individuals and organisations participating in the SSNAP, without whose efforts this study would not be possible. On behalf of everyone who has contributed to the project, we thank the clinical leads at each participating site and the individuals in the Royal College of Physicians Stroke Programme involved in coordinating data collection, whose names are available online (www.rcplondon.ac.uk/projects/outputs/ssnap-collaboration).

\section{References}

1 Aylin P. Making sense of the evidence for the "weekend effect". BMJ 2015; 351: h4652.

2 Bell CM, Redelmeier DA. Mortality among patients admitted to hospitals on weekends as compared with weekdays. N Engl J Med 2001; 345: 663-68.

3 Sorita A, Ahmed A, Starr SR, et al. Off-hour presentation and outcomes in patients with acute myocardial infarction: systematic review and meta-analysis. BMJ 2014; 348: f7393.

4 Ruiz M, Bottle A, Aylin PP. The Global Comparators project: international comparison of 30-day in-hospital mortality by day of the week. BMJ Qual Saf 2015; 24: 492-504.

5 NHS England. Everyone counts: planning for patients 2013/14. Dec 20, 2013. https://www.england.nhs.uk/wp-content/ uploads/2013/12/5yr-strat-plann-guid.pdf (accessed Oct 20, 2015).

6 Lilford RJ, Chen YF. The ubiquitous weekend effect: moving past proving it exists to clarifying what causes it. BMJ Qual Saf 2015; 24: $480-82$.

7 Lozano R, Naghavi M, Foreman K, et al. Global and regional mortality from 235 causes of death for 20 age groups in 1990 and 2010: a systematic analysis for the Global Burden of Disease Study 2010. Lancet 2012; 380: 2095-128.

8 Murray CJ, Vos T, Lozano R, et al. Disability-adjusted life years (DALYs) for 291 diseases and injuries in 21 regions, 1990-2010: a systematic analysis for the Global Burden of Disease Study 2010. Lancet 2012; 380: 2197-223.

9 Intercollegiate Stroke Working Party. National clinical guideline for stroke, 4th edn. Royal College of Physicians: London, 2012.

10 Guo G, Zhao H. Multilevel modeling for binary data. Ann Rev Socio 2000; 26: 441-62

11 van Swieten JC, Koudstaal PJ, Visser MC, Schouten HJ, van Gijn J. Interobserver agreement for the assessment of handicap in stroke patients. Stroke 1988; 19: 604-07.

12 Muller CJ, MacLehose RF. Estimating predicted probabilities from logistic regression: different methods correspond to different target populations. Int J Epidemiol 2014; 43: 962-70.
13 Rubin DB. Multiple imputation for nonresponse in surveys. New York, NY: Wiley, 1987.

14 Kostis WJ, Demissie K, Marcella SW, Shao YH, Wilson AC, Moyera AE. Weekend versus weekday admission and mortality from myocardial infarction. N Engl J Med 2007; 356: 1099-109.

15 Van-Hansen B, Riis AH, Sorensen HT, Christiansen CF. Out-of-hours and weekend admissions to Danish medical departments: admission rates and 30-day mortality for 20 common medical conditions. BMJ Open 2015; 11: e006731.

16 Coumbe A, John R, Kuskowski M, Agirbasli M, McFalls EO, Adabag S. Variation of mortality after coronary artery bypass surgery in relation to hour, day and month of the procedure. BMC Cardiovasc Dis 2011; 11: 63.

17 Magid DJ, Wang Y, Herrin J, et al. Relationship between time of day, day of week, timeliness of reperfusion, and in-hospital mortality for patients with acute ST-segment elevation myocardial infarction. JAMA 2005; 294: 803-12.

18 Turner N, Barber M, Dodds H, Dennis M, Langhorne P, Macleod MJ. Stroke patients admitted within normal working hours are more likely to achieve process standards and to have better outcomes J Neurol Neurosurg Psychiatry 2015; published online Aug 18. DOI:10.1136/jnnp-2015-311273.

19 Fang J, Saposnik G, Silver FL, Kapral MK. Investigators of the Registry of the Canadian Stroke Network. Association between weekend hospital presentation and fatality. Neurology 2010; 75: 1589-96.

20 Albright KC, Savitz SI, Raman R, et al. Comprehensive stroke centers and the 'weekend effect': the SPOTRIAS experience. Cerebrovasc Dis 2012; 34: 424-29.

21 McKinney JS, Deng Y, Kasner SE, Kostis JB, MIDAS 15 Study Group. Comprehensive stroke centers overcome the weekend versus weekday gap in stroke treatment and mortality. Stroke 2011; 42: 2403-09.

22 Bray BD, Ayis S, Campbell J, et al. Associations between stroke mortality and weekend working by stroke specialist physicians and registered nurses: prospective multicentre cohort study. PLoS Med 2014; 11: e1001705.

23 Coiera E, Wang Y, Magrabi F, Concha OP, Gallego B, Runciman W. Predicting the cumulative risk of death during hospitalization by modelling weekend, weekday and diurnal mortality risks. BMC Health Serv Res 2014; 14: 226.

24 Concha OP, Gallego B, Hillman K, Delaney GP, Coiera E. Do variations in hospital mortality patterns after weekend admission reflect reduced quality of care or different patient cohorts? A population-based study. BMJ Qual Saf 2014; 23: 215-22.

25 Lyndon A, Lee HC, Gay C, Gilbert WM, Gould JB, Lee KA. Effect of time of birth on maternal morbidity during childbirth hospitalization in California. Am J Obstet Gynecol 2015; 213: 705.e1-11.

26 Fonarow GC, Pan W, Saver JL, et al. Comparison of 30-day mortality models for profiling hospital performance in acute ischemic stroke with vs without adjustment for stroke severity. JAMA 2012; 308: 257-64

27 Meacock R, Doran T, Sutton M. What are the costs and benefits of providing comprehensive seven-day services for emergency hospital admissions? Health Econ 2015; 24: 907-12.

28 Emberson I, Lees KR, Lyden P, et al; Stroke Thrombolysis Trialists' Collaborative Group. Effect of treatment delay, age, and stroke severity on the effects of intravenous thrombolysis with alteplase for acute ischaemic stroke: a meta-analysis of individual patient data from randomised trials. Lancet 2014; 384: 1929-35.

29 Stroke Unit Trialists' Collaboration. Organised inpatient (stroke unit) care for stroke. Cochrane Database Syst Rev 2007; 4: CD000197. 